\title{
Distances to spaces of affine Baire-one functions
}

\author{
by \\ JiŘí SPURNÝ (Praha)
}

\begin{abstract}
Let $E$ be a Banach space and let $\mathcal{B}_{1}\left(B_{E^{*}}\right)$ and $\mathfrak{A}_{1}\left(B_{E^{*}}\right)$ denote the space of all Baire-one and affine Baire-one functions on the dual unit ball $B_{E^{*}}$, respectively. We show that there exists a separable $L_{1}$-predual $E$ such that there is no quantitative relation between $\operatorname{dist}\left(f, \mathcal{B}_{1}\left(B_{E^{*}}\right)\right)$ and $\operatorname{dist}\left(f, \mathfrak{A}_{1}\left(B_{E^{*}}\right)\right)$, where $f$ is an affine function on $B_{E^{*}}$. If the Banach space $E$ satisfies some additional assumption, we prove the existence of some such dependence.
\end{abstract}

1. Introduction. If $K$ is a compact (Hausdorff) space, we write $\mathcal{C}(K)$ for the space of all real-valued continuous functions on $K$ and $\mathcal{M}(K)$ for the space of all signed Radon measures on $K$. (By a Radon measure we mean a complete measure that is inner regular with respect to compact sets and is defined on a $\sigma$-algebra including all Borel subsets of $K$. A signed measure is Radon if the total variation $|\mu|$ of $\mu$ is a Radon measure. We refer the reader to [9, Section 416] for more information on Radon measures.) Let $\mathcal{M}^{1}(K)$ denote the set of all Radon probability measures on $K$. We always consider $\mathcal{M}(K)$ endowed with the weak* topology. We say that a function $f: K \rightarrow \mathbb{R}$ is universally measurable if $f$ is $\mu$-measurable for every $\mu \in \mathcal{M}^{1}(K)$. We denote the space of all bounded universally measurable functions on $K$ by $\mathcal{U}^{b}(K)$.

If $X$ is a compact convex subset of a real locally convex space, let $\mathfrak{A}^{b}(X)$ and $\mathfrak{A}^{c}(X)$ denote the spaces of all bounded affine functions on $X$ and continuous affine functions on $X$, respectively. Any $\mu \in \mathcal{M}^{1}(X)$ has its unique barycenter $r(\mu) \in X$, i.e., the point $x \in X$ satisfying $f(x)=\mu(f)$ for any $f \in \mathfrak{A}^{c}(X)$ (see [1, Proposition I.2.1]). We sometimes say that $\mu$ represents $x$. A function $f: X \rightarrow \mathbb{R}$ is strongly affine (or satisfies the barycentric formula) if $f$ is universally measurable, $\mu(f)$ exists and $f(r(\mu))=\mu(f)$ for any $\mu \in \mathcal{M}^{1}(X)$. We write $\mathfrak{A}_{\mathrm{bf}}(X)$ for the space of all strongly affine functions on $X$ (i.e. functions satisfying the barycentric formula) and recall that

2010 Mathematics Subject Classification: Primary 46B99; Secondary 52A07.

Key words and phrases: $L_{1}$-predual, affine function, Baire-one function, fragmented function, simplex. 
it is easy to see that any strongly affine function is affine and bounded (see the proof of [13, Satz 2.1(c)]).

By a result of B. Cascales, W. Marciszewski and M. Raja [5, Proposition 4.1], $\operatorname{dist}(f, \mathcal{C}(X))=\operatorname{dist}\left(f, \mathfrak{A}^{c}(X)\right)$ for any $f \in \mathfrak{A}^{b}(X)$. If $E$ is a Banach space, its dual unit ball $B_{E^{*}}$ endowed with the weak* topology is an example of a compact convex set. Given an element $x^{* *} \in E^{* *}$, let $f$ denote its restriction to $B_{E^{*}}$. By the fact above, $\operatorname{dist}\left(f, \mathcal{C}\left(B_{E^{*}}\right)\right)=\operatorname{dist}\left(f, \mathfrak{A}^{c}\left(B_{E^{*}}\right)\right)$ (see [5, Corollary 4.2]).

As a further step, a paper [2] by C. Angosto, B. Cascales and I. Namioka investigates how to measure distance of a function to the space of Baire-one functions. Let us recall that, given two topological spaces $K$ and $E$, the space $\mathcal{B}_{1}(K, E)$ consists of all mappings $f: K \rightarrow E$ that can be obtained as the pointwise limit of a sequence of continuous mappings from $K$ to $E$. If $E=\mathbb{R}$, we write $\mathcal{B}_{1}(K)$ for $\mathcal{B}_{1}(K, \mathbb{R})$. If $f: K \rightarrow E$ is a mapping from a topological space $K$ to a metric space $E, f$ is said to be $\varepsilon$-fragmented if for any closed set $F \subset K$ there exists a relatively open nonempty subset $U$ of $F$ such that $\operatorname{diam} f(U)<\varepsilon$ (see [2, p. 105]). Then $\operatorname{frag}(f)$ is defined as

$$
\operatorname{frag}(f)=\inf \{\varepsilon>0: f \text { is } \varepsilon \text {-fragmented }\}
$$

if such an $\varepsilon>0$ exists, and frag $(f)=\infty$ otherwise. If $f: K \rightarrow \mathbb{R}$ is a function on a metrizable compact space, it follows from [2, Corollary 2.6] that $\operatorname{dist}\left(f, \mathcal{B}_{1}(K)\right)=\frac{1}{2} \operatorname{frag}(f)$.

If $X$ is a compact convex set, let $\mathfrak{A}_{1}(X)$ stand for the space of all pointwise limits of sequences of functions from $\mathfrak{A}^{c}(X)$. By [20, Théorème 80] (see also [7, p. 611]), $\mathcal{B}_{1}(X) \cap \mathfrak{A}^{b}(X)=\mathfrak{A}_{1}(X)$, and any function in $\mathfrak{A}_{1}(X)$ is a pointwise limit of a bounded sequence in $\mathfrak{A}^{c}(X)$. If $f \in \mathfrak{A}^{b}(X)$, following the result on continuous functions we might ask whether $\operatorname{dist}\left(f, \mathcal{B}_{1}(X)\right)=$ $\operatorname{dist}\left(f, \mathfrak{A}_{1}(X)\right)$. The aim of our paper is to present an example that disproves this. (We recall that a Banach space is an $L_{1}$-predual if its dual is isometric to a space $L^{1}(\mu)$ for a suitable measure $\mu$; see [7, p. 625].)

THEOREM 1.1. There exists a separable $L_{1}$-predual $E$ with the following property: for any $\varepsilon>0$ there exists $x^{* *} \in B_{E^{* *}}$ such that the function $f=\left.x^{* *}\right|_{B_{E^{*}}}$ satisfies

- $f$ is strongly affine,

- $\operatorname{dist}\left(f, \mathcal{B}_{1}\left(B_{E^{*}}\right)\right)<\varepsilon$,

- $\operatorname{dist}\left(f, \mathfrak{A}_{1}\left(B_{E^{*}}\right)\right) \geq 1 / 2$.

If an $L_{1}$-predual $E$ satisfies an additional topological condition imposed on the set ext $B_{E^{*}}$ of all extreme points of its dual unit ball $B_{E^{*}}$, we obtain a quantitative relation between the distance to Baire-one functions and the distance to affine Baire-one functions. We recall that a subset $H$ of a topological space $K$ is said to be an $H$-set (or a resolvable set) if the char- 
acteristic function $\chi_{H}$ satisfies frag $\left(\chi_{H}\right)=0$ (see [14, §12]). We recall that a mapping $f: K \rightarrow E$ between two topological spaces is Baire measurable if $f^{-1}(U)$ is a Baire subset of $K$ for any $U \subset E$ open.

TheOREM 1.2. Let $E$ be an $L_{1}$-predual such that the set of extreme points of the dual unit ball is a Lindelöf $H$-set in the weak $k^{*}$ topology. Let $x^{* *} \in E^{* *}$ and $f=\left.x^{* *}\right|_{B_{E^{*}}}$. If

- E is separable, or

- $f$ is Baire measurable,

then $\operatorname{dist}\left(f, \mathfrak{A}_{1}\left(B_{E^{*}}\right)\right) \leq 5 \operatorname{dist}\left(f, \mathcal{B}_{1}\left(B_{E^{*}}\right)\right)$.

We remark that, for a separable space $E$, the topological condition imposed on ext $B_{E^{*}}$ is equivalent to ext $B_{E^{*}}$ being of type $F_{\sigma}$. This can be seen from the following two facts: a subset of a compact metrizable space is an $H$-set if and only if it is both of type $F_{\sigma}$ and $G_{\delta}$ (use $[14, \S 26, \mathrm{X}]$ and the Baire category theorem); the set of extreme points in a metrizable compact convex set is of type $G_{\delta}$ (see [1, Corollary I.4.4]).

We also point out that the topological assumption in Theorem 1.2 is satisfied when ext $B_{E^{*}}$ is an $F_{\sigma}$ set. To see this, we first notice that ext $B_{E^{*}}$ is then a Lindelöf space. Second, we need to check that ext $B_{E^{*}}$ is an $H$-set in $B_{E^{*}}$. To this end, assume that $F \subset B_{E^{*}}$ is a nonempty closed set such that both $F \cap \operatorname{ext} B_{E^{*}}$ and $F \backslash \operatorname{ext} B_{E^{*}}$ are dense in $F$. By [25, Théorème 2], we can write

$$
\operatorname{ext} B_{E^{*}}=\bigcap_{n=1}^{\infty}\left(H_{n} \cup V_{n}\right),
$$

where $H_{n} \subset B_{E^{*}}$ is closed and $V_{n} \subset B_{E^{*}}$ is open, $n \in \mathbb{N}$. Thus both $F \backslash \operatorname{ext} B_{E^{*}}$ and $F \cap \operatorname{ext} B_{E^{*}}$ are comeager disjoint sets in $F$, contradicting the Baire category theorem. Hence ext $B_{E^{*}}$ is an $H$-set.

The following result presents a condition of a different type that still yields a conclusion similar to that of Theorem 1.2 .

TheOREM 1.3. Let $E$ be a Banach space not containing $\ell^{1}, x^{* *} \in E^{* *}$ and let $f=\left.x^{* *}\right|_{B_{E^{*}}}$. Then $\operatorname{dist}\left(f, \mathfrak{A}_{1}\left(B_{E^{*}}\right)\right) \leq 2 \operatorname{dist}\left(f, \mathcal{B}_{1}\left(B_{E^{*}}\right)\right)$.

If $E$ above is assumed to be separable, any element $x^{* *} \in E^{* *}$ is in $\mathfrak{A}_{1}\left(B_{E^{*}}\right)$ when restricted to $B_{E^{*}}$ (see [18] or [3], Theorem II.1.3]) and thus the inequality is vacuously satisfied (the author would like to thank M. Raja for this important remark).

We also present a variant of [2, Theorem 2.5] for nonmetrizable compact spaces needed for our purposes.

TheOREM 1.4. Let $K$ be a compact space and $f: K \rightarrow E$ be a function from $K$ to a Banach space $E$. 
(a) $\frac{1}{2} \operatorname{frag}(f) \leq \operatorname{dist}\left(f, \mathcal{B}_{1}(K, E)\right)$.

(b) If $f$ is Baire measurable, then $\operatorname{dist}\left(f, \mathcal{B}_{1}(K, E)\right) \leq \operatorname{frag}(f)$.

(c) If $f$ is Baire measurable and $E=\mathbb{R}$, then $\operatorname{dist}\left(f, \mathcal{B}_{1}(K)\right)=\frac{1}{2} \operatorname{frag}(f)$.

Our construction of a separable $L_{1}$-predual in Theorem 1.1 is based upon the notion of a simplicial function space. We recall that, given a compact space $K$, a function space $\mathcal{H}$ is a subspace of $\mathcal{C}(K)$ that contains constants and separates points of $K$. We use the construction from [23] to get the desired example of Theorem 1.1.

Throughout, we follow the notation and definitions from [23].

We just recall that, given a function space $\mathcal{H}$ on a compact space $K$, the state space $\mathbf{S}(\mathcal{H})$ of $\mathcal{H}$ is defined as

$$
\mathbf{S}(\mathcal{H})=\left\{s \in \mathcal{H}^{*}:\|s\|=s(1)=1\right\} .
$$

If $\mathbf{S}(\mathcal{H})$ is endowed with the weak ${ }^{*}$ topology, it is a compact convex set. The space $K$ is homeomorphically embedded into $\mathbf{S}(\mathcal{H})$ via the evaluation mapping $\phi: K \rightarrow \mathbf{S}(\mathcal{H})$ defined by

$$
\phi(x)(h)=h(x), \quad h \in \mathcal{H}, x \in K .
$$

We denote

$$
\mathcal{U}^{b}(K) \cap \mathcal{H}^{\perp \perp}=\left\{f \in \mathcal{U}^{b}(K): \mu(f)=0 \text { for all } \mu \in \mathcal{H}^{\perp}\right\}
$$

and $X=\mathbf{S}(\mathcal{H})$. Let $\pi: \mathcal{M}^{1}(K) \rightarrow X$ denote the restriction mapping. It follows from [23, Theorem 2.5] that the formula

$$
\text { If }(s)=\mu(f), \quad \pi(\mu)=s, s \in X, \quad f \in \mathcal{U}^{b}(K) \cap \mathcal{H}^{\perp \perp},
$$

defines an isometric isomorphism $I: \mathcal{U}^{b}(\mathcal{K}) \cap \mathcal{H}^{\perp \perp} \rightarrow \mathfrak{A}_{\mathrm{bf}}(X)$. Moreover,

$$
I\left(\mathcal{B}_{\alpha}^{b}(K) \cap \mathcal{H}^{\perp \perp}\right)=\mathcal{B}_{\alpha}^{b}(X) \cap \mathfrak{A}_{\mathrm{bf}}(X), \quad \alpha \in\left[0, \omega_{1}\right) .
$$

To illuminate relations between compact convex sets and Banach spaces, let us recall the following facts. If $X$ is a compact convex set and $E=\mathfrak{A}^{c}(X)$, the state space $\mathbf{S}\left(\mathfrak{A}^{c}(X)\right)$ is affinely homeomorphic to $X$ via the evaluation mapping $\phi$. The dual unit ball $B_{E^{*}}$ equals $\operatorname{co}(\phi(X) \cup-\phi(X))$ and the weak topology on $E$ coincides with the topology of pointwise convergence on $\mathfrak{A}^{c}(X)$. Any function $f \in \mathfrak{A}^{b}(X)$ has a unique extension to $E^{*}=\operatorname{span} \phi(X)$. This provides an identification of $E^{* *}$ with $\mathfrak{A}^{b}(X)$. Moreover, the weak* topology on $E^{* *}$ coincides with the topology of pointwise convergence on $\mathfrak{A}^{b}(X)$.

A compact convex set $X$ is a simplex if $\mathfrak{A}^{c}(X)$ is an $L_{1}$-predual (for more information on simplices, see [1, Chapter II, §3], [4, Section 2.7], [6. Chapter 6, §28], [7, Section 3], [15, Chapter 7, §20], [19, Chapter 10] or [21, Chapter 6, §23]).

If $E$ is a Banach space, a function $f: B_{E^{*}} \rightarrow \mathbb{R}$ is the restriction of an element of $E$ if and only if $f \in \mathfrak{A}^{c}\left(B_{E^{*}}\right)$ and $f(0)=0$. 
2. Construction of function spaces. We use the construction of a function space from [23, Section 5]. For a fixed natural number $m>1$, let $\mathcal{H}_{0}, \ldots, \mathcal{H}_{m}$ be the simplicial function spaces on the metrizable compact spaces $K_{0}, \ldots, K_{m}$ constructed in [23, Inductive construction 5.2]. Let $\left\{F_{s}: s \in \mathbb{N}^{<\mathbb{N}}\right\}$ and $\mathcal{F}_{n}=\left\{F_{n}(k): k \in \mathbb{N}\right\}, n=0, \ldots, m$, be the objects defined there.

We recall from [23, Lemma 3.3] that $\left\{F_{s}: s \in \mathbb{N}<\mathbb{N}\right\}$ is a family of sets in $K_{0}=[0,1]$ with the following properties:

(a) $F_{\emptyset}=K_{0}$,

(b) $\left\{F_{s^{\wedge} n}: n \in \mathbb{N}\right\}$ is a disjoint family of nonempty nowhere dense perfect subsets of $F_{s}$,

(c) $\bigcup\left\{F_{s^{\wedge} n}: n \in \mathbb{N}\right\}$ is dense in $F_{s}$,

(d) $\operatorname{diam} F_{s}<2^{-\left(s_{1}+\cdots+s_{|s|}\right)}, s \in \mathbb{N}^{<\mathbb{N}}$.

We further demand that the family $\left\{F_{s}: s \in \mathbb{N}^{<\mathbb{N}}\right\}$ satisfies the following stronger version of $(\mathrm{c})$ :

(e) both $\left\{F_{s^{\wedge} n}: n\right.$ odd $\}$ and $\left\{F_{s^{\wedge} n}: n\right.$ even $\}$ are dense in $F_{s}, s \in \mathbb{N}<\mathbb{N}$.

This family is used in [23, Inductive construction 5.2] for an inductive construction of function spaces with increasing complexity. Roughly speaking, the construction proceeds as follows.

We take the sets of the first level in $K_{0}$, i.e., $\left\{F_{n}: n \in \mathbb{N}\right\}$, and create a new compact space $K_{1}$ by adding to $K_{0}$ two copies of each $F_{n}$. We imagine each point of $F_{n}$ to be the average of two points "above" and "below" and encode it in the definition of the new function space $\mathcal{H}_{1}$ on $K_{1}$. For each set $F_{n}$, we consider the sets of the second level $\left\{F_{n^{\wedge} k}: k \in \mathbb{N}\right\}$ and transfer them into the just created copies of $F_{n}$. These new sets form the family $\mathcal{F}_{1}$.

The second step splits up the sets from $\mathcal{F}_{1}$ and transfers the sets $\left\{F_{s}\right.$ : $|s|=3\}$ of the third level into them. Proceeding inductively, we create function spaces $\mathcal{H}_{m}$ on compact spaces $K_{m}, m=0,1, \ldots$

LEMMA 2.1. The following asertions hold:

(a) $\mathcal{H}_{m}$ is a simplicial function space with $\mathcal{A}_{c}\left(\mathcal{H}_{m}\right)=\mathcal{H}_{m}$,

(b) $K_{m} \backslash \mathrm{Ch}_{\mathcal{H}_{m}} K_{m}=\bigcup_{n=0}^{m-1} \bigcup \mathcal{F}_{n}$.

Proof. Assertion (a) follows by inductive use of [23, Lemma 5.1(c),(e)], and (b) from [23, Lemma 5.1(d)].

Let $\delta=(2 m+1)^{-1}$.

Definition 2.2. We define inductively a function $f_{m}: K_{m} \rightarrow[-1,1]$ such that $f_{m}$ is constant on each element of $\mathcal{F}_{n}$ for every $n \in\{0, \ldots, m\}$. The definition is as follows: 
- For $x \in K_{0}$, we set

$$
f_{m}(x)= \begin{cases}\delta, & x \in F_{s}, s \in \mathbb{N} \text { is odd } \\ -\delta, & x \in K_{0} \backslash \bigcup\left\{F_{s}: s \in \mathbb{N} \text { is odd }\right\} .\end{cases}
$$

- Assume that $f_{m}$ is defined on each $K_{0}, \ldots, K_{n}$ for some $n \in\{0, \ldots$, $m-1\}$. Let $\mathcal{F}_{n}=\left\{F_{n}(k): k \in \mathbb{N}\right\}$ be the enumeration of the family $\mathcal{F}_{n}$ and let $a_{n}(k)$ be the value of $f_{m}$ on $F_{n}(k)$. Let

$$
F(s, k,+), F(s, k,-), \quad k \in \mathbb{N}, s \in \mathbb{N}^{n+2},
$$

be as in equations (8) of [23, Inductive construction 5.2]. Let $\mathbb{N}_{\text {odd }}^{n+2}$ denote the set of all sequences $s \in \mathbb{N}^{n+2}$ with $s_{n+2}$ odd. Then we define the function $f_{m}$ for

$$
x \in K_{n+1} \backslash K_{n}=\bigcup_{k=1}^{\infty}\left(F_{n}(k) \times\{1 / k\}\right) \cup\left(F_{n}(k) \times\{-1 / k\}\right)
$$

as

$$
\begin{aligned}
& f_{m}(x) \\
& = \begin{cases}a_{n}(k)+2 \delta, & x \in \bigcup\left\{F(s, k,+): s \in \mathbb{N}_{\text {odd }}^{n+2}\right\}, \\
a_{n}(k), & x \in\left(F_{n}(k) \times\{1 / k\}\right) \backslash \bigcup\left\{F(s, k,+): s \in \mathbb{N}_{\text {odd }}^{n+2}\right\}, \\
a_{n}(k)-2 \delta, & x \in \bigcup\left\{F(s, k,-): s \in \mathbb{N}_{\text {odd }}^{n+2}\right\}, \\
a_{n}(k), & x \in\left(F_{n}(k) \times\{-1 / k\}\right) \backslash \bigcup\left\{F(s, k,-): s \in \mathbb{N}_{\text {odd }}^{n+2}\right\} .\end{cases}
\end{aligned}
$$

Lemma 2.3. The function $f_{m}$ from Definition 2.2 has the following properties:

(a) $f_{m}\left(K_{m}\right) \subset[-1,1]$,

(b) $\operatorname{frag}\left(f_{m}\right)=2 \delta$,

(c) $f_{m} \in \mathcal{B}_{2}^{b}\left(K_{m}\right) \cap \mathcal{H}_{m}^{\perp \perp}$,

(d) $\operatorname{dist}\left(f_{m}, \mathcal{B}_{1}^{b}\left(K_{m}\right) \cap \mathcal{H}_{m}^{\perp \perp}\right) \geq 1 / 2$.

Proof. To verify (a), we notice that Definition 2.2 yields the following fact: The greatest value of $f_{m}$ is $\delta+2 \delta m=1$ and the least value of $f_{m}$ is $-\delta-2 \delta m=-1$.

To prove (b), we note that $K_{m}$ can be written as

$$
K_{m}=K_{0} \cup \bigcup_{n=1}^{m}\left(K_{n} \backslash K_{n-1}\right) .
$$

We show that $\left.f_{m}\right|_{K_{0}}$ and $\left.f_{m}\right|_{K_{n} \backslash K_{n-1}}, n=1, \ldots, m$, are $2 \delta$-fragmented. Obviously, $\left.f_{m}\right|_{K_{0}}$ is $2 \delta$-fragmented. If $n \in\{1, \ldots, m\}, K_{n} \backslash K_{n-1}$ can be written as a countable union of clopen subsets of $K_{n}$ such that the restriction of $f_{m}$ to each of them is $2 \delta$-fragmented. Let $F \subset K_{m}$ be a closed set and $\varepsilon>2 \delta$. If $F \subset K_{0}$, it is easy to find a relatively open subset of $F$ with $\operatorname{diam} f_{m}(U)<\varepsilon$. Otherwise we find the greatest index $n \in\{1, \ldots, m\}$ such 
that $F \cap\left(K_{n} \backslash K_{n-1}\right) \neq \emptyset$. Since $K_{n} \backslash K_{n-1}$ is an open subset of $K_{n-1}$, there exists a relatively open subset $U$ of $F$ with $\operatorname{diam} f_{m}(U)<\varepsilon$. Hence assertion (b) follows.

We start the proof of (c) by observing that it is enough to show that the restriction of $f_{m}$ to any member of the partition from (2.1) is a Baire-two function. This is easy on $K_{0}$ and, as above, we find that every $K_{n} \backslash K_{n-1}$, $n=1, \ldots, m$, is a countable union of clopen subsets of $K_{n}$ and that the restriction of $f_{m}$ to each member of this family is a Baire-two function.

For the second part of (c), the function $f_{m}$ is in $\mathcal{A}\left(\mathcal{H}_{m}\right)$ by inductive use of [23, Lemma 5.1(f)]. Indeed, $\left.f_{m}\right|_{K_{0}} \in \mathcal{A}\left(\mathcal{H}_{0}\right)$ obviously. By Definition [2.2, $\left.f_{m}\right|_{K_{1}}$ satisfies equations (6) of [23, Key step 5.1], and thus $\left.f_{m}\right|_{K_{1}} \in \mathcal{A}\left(\mathcal{H}_{1}\right)$ by [23, Lemma 5.1(f)]. Proceeding inductively, we verify that $\left.f_{m}\right|_{K_{n}} \in \mathcal{A}\left(\mathcal{H}_{n}\right)$ for every $n \in\{0, \ldots, m\}$. Since $\mathcal{H}_{m}$ is simplicial, [23, Theorem 2.6(b2)] yields

$$
f \in\left(\mathcal{A}_{c}\left(\mathcal{H}_{m}\right)\right)^{\perp \perp}=\mathcal{H}_{m}^{\perp \perp} .
$$

To show (d), let $g \in \mathcal{B}_{1}^{b}\left(K_{m}\right) \cap \mathcal{H}_{m}^{\perp \perp}$ be arbitrary. We fix $\varepsilon \in(0, \delta)$ and inductively find $F_{n} \in \mathcal{F}_{n}, n=0, \ldots, m$, such that

- $\left|g-f_{m}\right|>(n+1) \delta-3^{n} \varepsilon$ on $F_{n}, n=0, \ldots, m$.

For $n=0$, we find $x \in K_{0}$ such that $\left.g\right|_{K_{0}}$ is continuous at $x_{0}$ (see [14, $\S 27, \mathrm{X}])$. Let $U \subset K_{0}$ be a neighborhood of $x$ such that diam $g(U)<\varepsilon$. It follows from properties (d) and (e) of the system $\mathcal{F}_{0}$ and from Definition 2.2 that there exist $F, F^{\prime} \in \mathcal{F}_{0}$ such that $F \cup F^{\prime} \subset U$ and $f_{m}=\delta$ on $F$ and $f_{m}=-\delta$ on $F^{\prime}$. Hence it follows, by distinguishing the cases $g(x) \leq 0$ and $g(x) \geq 0$, that there exists $F_{0} \in \mathcal{F}_{0}$ such that $\left|g-f_{m}\right|>\delta-\varepsilon$ on $F_{0}$.

Assume now that the construction has been completed up to the $n$th step for some $n \in\{0, \ldots, m-1\}$. Hence we have $F_{n} \in \mathcal{F}_{n}$ such that

$$
\left|g-f_{m}\right|>(n+1) \delta-3^{n} \varepsilon \quad \text { on } F_{n} .
$$

Let $k \in \mathbb{N}$ be the index of $F_{n}$ in $\mathcal{F}_{n}$; that is, $F_{n}=F_{n}(k)$. Let $a_{n}(k)$ denote the value of $f_{m}$ on $F_{n}(k)$. Then

$$
\left(F_{n}(k) \times\{1 / k\}\right) \cup\left(F_{n}(k) \times\{-1 / k\}\right) \subset K_{n+1} \subset K_{m} .
$$

We find $x=(x, 0) \in F_{n}(k)$ such that $(x, 1 / k)$ is a point of continuity of the function $\left.g\right|_{F_{n}(k) \times\{1 / k\}}$.

CASE 1. Assume first that

$$
g(x, 1 / k) \in\left(-\infty, a_{n}(k)-n \delta\right) \cup\left(a_{n}(k)+(n+2) \delta, \infty\right) .
$$

If $g(x, 1 / k) \in\left(-\infty, a_{n}(k)-n \delta\right)$, we find a neighborhood $U$ of $x$ in $F_{n}(k)$ such that the same holds for all elements of $U \times\{1 / k\}$. By Definition 2.2 and properties of $\mathcal{F}_{n+1}$ described above, there exists a set $F_{n+1} \in \mathcal{F}_{n+1}$ such 
that $F_{n+1} \subset U \times\{1 / k\}$ and $f_{m}-g=a_{n}(k)+2 \delta-g>a_{n}(k)+2 \delta-\left(a_{n}(k)-n \delta\right)=(n+2) \delta \quad$ on $F_{n+1}$.

Analogously, if $g(x, 1 / k) \in\left(a_{n}(k)+(n+2) \delta, \infty\right)$, again we find a neighborhood $U$ of $x$ in $F_{n}(k)$ such that the same holds for all elements of $U \times\{1 / k\}$. By Definition 2.2 and properties (d) and (e) of $\mathcal{F}_{n+1}$, there exists a set $F_{n+1} \in \mathcal{F}_{n+1}$ such that $F_{n+1} \subset U \times\{1 / k\}$ and

$$
g-f_{m}=g-a_{n}(k)>a_{n}(k)+(n+2) \delta-a_{n}(k)=(n+2) \delta \quad \text { on } F_{n+1} .
$$

This finishes the inductive step in this case.

Case 2. Assume now that

$$
g(x, 1 / k) \in\left[a_{n}(k)-n \delta, a_{n}(k)+(n+2) \delta\right] .
$$

Let $U$ be a neighborhood of $x$ in $F_{n}(k)$ such that

$$
-3^{n} \varepsilon+a_{n}(k)-n \delta<g<a_{n}(k)+(n+2) \delta+3^{n} \varepsilon \quad \text { on } U \times\{1 / k\} .
$$

Let $y=(y, 0) \in U$ be such that $(y,-1 / k)$ is a point of continuity of $\left.g\right|_{F_{n}(k) \times\{-1 / k\}}$. We see from $(2.2)$ that

$$
\left|g(y, 0)-f_{m}(y, 0)\right|>(n+1) \delta-3^{n} \varepsilon .
$$

CAse 2a. Assume first that

$$
g(y, 0)<f_{m}(y, 0)-(n+1) \delta+3^{n} \varepsilon=a_{n}(k)-(n+1) \delta+3^{n} \varepsilon .
$$

Since $g$ is $\mathcal{H}_{m}$-affine, [23, equations (6) in Key step 5.1] yield

$$
\begin{aligned}
g(y,-1 / k) & =2 g(y, 0)-g(y, 1 / k) \\
& <2 a_{n}(k)-2(n+1) \delta+2 \cdot 3^{n} \varepsilon-\left(-3^{n} \varepsilon+a_{n}(k)-n \delta\right) \\
& =a_{n}(k)-(n+2) \delta+3^{n+1} \varepsilon .
\end{aligned}
$$

By the continuity of $\left.g\right|_{F_{n}(k) \times\{-1 / k\}}$ at $(y,-1 / k)$, there exists a neighborhood $V$ of $y$ in $F_{n}(k)$ such that $V \subset U$ and

$$
g<a_{n}(k)-(n+2) \delta+3^{n+1} \varepsilon \quad \text { on } V \times\{-1 / k\} .
$$

By properties of $\mathcal{F}_{n+1}$ and Definition 2.2, there exists $F_{n+1} \in \mathcal{F}_{n+1}$ such that $F_{n+1} \subset V \times\{-1 / k\}$ and

$$
g<a_{n}(k)-(n+2) \delta+3^{n+1} \varepsilon=f_{m}-(n+2) \delta+3^{n+1} \varepsilon \quad \text { on } F_{n+1} .
$$

This finishes the inductive step in this case.

CASE 2b. If

$$
g(y, 0)>f_{m}(y, 0)+(n+1) \delta-3^{n} \varepsilon=a_{n}(k)+(n+1) \delta-3^{n} \varepsilon,
$$

[23, equations (6) in Key step 5.1] give

$$
\begin{aligned}
g(y,-1 / k) & =2 g(y, 0)-g(y, 1 / k) \\
& >2 a_{n}(k)+2(n+1) \delta-2 \cdot 3^{n} \varepsilon-\left(a_{n}(k)+(n+2) \delta+3^{n} \varepsilon\right) \\
& =a_{n}(k)+n \delta-3^{n+1} \varepsilon .
\end{aligned}
$$


By the continuity of $\left.g\right|_{F_{n}(k) \times\{-1 / k\}}$ at $(y,-1 / k)$, there exists a neighborhood $V$ of $y$ in $F_{n}(k)$ such that $V \subset U$ and

$$
g>a_{n}(k)+n \delta-3^{n+1} \varepsilon \quad \text { on } V \times\{-1 / k\} .
$$

By properties of $\mathcal{F}_{n+1}$ and Definition 2.2, there exists $F_{n+1} \in \mathcal{F}_{n+1}$ such that $F_{n+1} \subset V \times\{-1 / k\}$ and

$$
g>a_{n}(k)+n \delta-3^{n+1} \varepsilon=f_{m}+(n+2) \delta-3^{n+1} \varepsilon \quad \text { on } F_{n+1} .
$$

The inductive step is finished also in this case.

After the $m$ th step of the construction we obtain a set $F_{m} \in \mathcal{F}_{m}$ such that

$$
\left|g-f_{m}\right|>(m+1) \delta-3^{m} \varepsilon \quad \text { on } F_{m} .
$$

Thus

$$
\left\|g-f_{m}\right\|>(m+1) \delta-3^{m} \varepsilon=\frac{m+1}{2 m+1}-3^{m} \varepsilon \geq \frac{1}{2}-3^{m} \varepsilon .
$$

Since $\varepsilon \in(0, \delta)$ is arbitrary, $\left\|g-f_{m}\right\| \geq 1 / 2$. Hence $\operatorname{dist}\left(f_{m}, \mathcal{B}_{1}^{b}\left(K_{m}\right) \cap \mathcal{H}_{m}^{\perp \perp}\right)$ $\geq 1 / 2$.

\section{Auxiliary results}

Lemma 3.1. Let $\varphi: X \rightarrow Y$ be a continuous surjection of a compact space $X$ onto a compact space $Y$ and let $g: Y \rightarrow Z$ be a function from $Y$ to a metric space $(Z, \rho)$. Then $\operatorname{frag}(g)=\operatorname{frag}(g \circ \varphi)$.

Proof. If $\operatorname{frag}(g)=\infty$, then $\operatorname{frag}(g \circ \varphi) \leq \operatorname{frag}(g)$. Assume that $\operatorname{frag}(g)<\infty$ and let $\varepsilon>0$ be such that $g$ is $\varepsilon$-fragmented. If $F \subset X$ is a nonempty closed set, let $W \subset Y$ be an open set intersecting $\varphi(F)$ such that $\operatorname{diam} g(W \cap \varphi(F))<\varepsilon$. Then $\operatorname{diam}(g \circ \varphi)\left(F \cap \varphi^{-1}(W)\right)<\varepsilon$, and thus $\operatorname{frag}(g \circ \varphi) \leq \operatorname{frag}(g)$.

To prove the opposite inequality, assume that $\operatorname{frag}(g \circ \varphi)<\infty$. Let $\varepsilon>0$ be such that $g \circ \varphi$ is $\varepsilon$-fragmented and let $H \subset Y$ be a nonempty closed set. Using compactness and Zorn's lemma, we find a closed set $F \subset X$ such that $\varphi(F)=H$ and $F$ is a closed set which is a minimal set (with respect to inclusion) with this property. Let $U \subset X$ be an open set intersecting $F$ with $\operatorname{diam}(g \circ \varphi)(U \cap F)<\varepsilon$. Then $H \backslash \varphi(F \backslash U)$ is a nonempty relatively open subset of $H$ (it is nonempty by the minimality of $F$ ) satisfying

$$
\operatorname{diam} g(H \backslash \varphi(F \backslash U))<\varepsilon .
$$

Hence $\operatorname{frag}(g) \leq \operatorname{frag}(g \circ \varphi)$, which concludes the proof.

Lemma 3.2. Let $K$ be a metrizable compact space and let $f \in \mathcal{U}^{b}(K)$. If $\widehat{f}: B_{\mathcal{M}(K)} \rightarrow \mathbb{R}$ is defined as $\widehat{f}(\mu)=\mu(f), \mu \in B_{\mathcal{M}(K)}$, then $\operatorname{frag}(f)=$ $\operatorname{frag}(\widehat{f})$. 
Proof. Let $\varepsilon>\frac{1}{2} \operatorname{frag}(f)$ be arbitrary. Using [2, Corollary 2.6], we find a function $g \in \mathcal{B}_{1}(K)$ such that $\|f-g\|<\varepsilon$. Without loss of generality we may assume that $\|g\|=\|f\|$. If $\widehat{g}: B_{\mathcal{M}(K)} \rightarrow \mathbb{R}$ is defined as

$$
\widehat{g}(\mu)=\mu(g), \quad \mu \in B_{\mathcal{M}(K)},
$$

then $\|\widehat{f}-\widehat{g}\|<\varepsilon$. Hence $\operatorname{dist}\left(\widehat{f}, \mathcal{B}_{1}\left(B_{\mathcal{M}(K)}\right)\right)<\varepsilon$, and thus $\frac{1}{2} \operatorname{frag}(\widehat{f})<\varepsilon$. It follows that $\operatorname{frag}(\widehat{f}) \leq \operatorname{frag}(f)$. Since the opposite inequality is obvious, the proof is complete.

Lemma 3.3. If $\mathcal{H}$ is a function space on a metrizable compact space $K$ and $f \in \mathcal{U}^{b}(K) \cap \mathcal{H}^{\perp \perp}$, then $\operatorname{frag}(f)=\operatorname{frag}(I f)$.

Proof. Let $\varepsilon>\operatorname{frag}(f)$ be arbitrary. If $\widehat{f}: \mathcal{M}^{1}(K) \rightarrow \mathbb{R}$ is defined as

$$
\widehat{f}(\mu)=\mu(f), \quad \mu \in \mathcal{M}^{1}(K),
$$

then $\operatorname{frag}(\widehat{f})<\varepsilon$ by Lemma 3.2. Since $\pi: \mathcal{M}^{1}(K) \rightarrow \mathbf{S}(\mathcal{H})$ is a continuous surjection, Lemma 3.1 gives frag $(I f)<\varepsilon$. Since $\varepsilon>\operatorname{frag}(f)$ is arbitrary, $\operatorname{frag}(I f) \leq \operatorname{frag}(f)$.

The opposite inequality follows from the fact that $I f \circ \phi=f$.

The following fact is a variant of the argument in [19, p. 88].

Lemma 3.4. Let $f: X \rightarrow \mathbb{R}$ be a convex function on a compact convex set $X$ such that $\operatorname{frag}(f)<\infty$. Then $f$ is lower bounded.

Proof. Without loss of generality we may assume that $0 \in X$. Assume that there exists a sequence $\left\{x_{n}\right\}$ of points in $X$ such that $f\left(x_{n}\right) \rightarrow-\infty$. We consider the set

$$
S=\left\{\lambda \in \ell^{1}: \sum_{n=1}^{\infty} \lambda(n) \leq 1, \lambda(n) \geq 0 \text { for each } n \in \mathbb{N}\right\}
$$

with the weak* topology (as usual, the space $\ell^{1}$ is identified with the dual space of $c_{0}$ ) and a mapping $\varphi: S \rightarrow X$ defined by

$$
\varphi(\lambda)=\sum_{n=1}^{\infty} \lambda(n) x_{n}, \quad \lambda \in S .
$$

Then $\varphi$ is a continuous affine mapping and, by Lemma 3.1 .

$$
\operatorname{frag}(f \circ \varphi)=\operatorname{frag}\left(\left.f\right|_{\varphi(S)}\right) \leq \operatorname{frag}(f)=\eta<\infty .
$$

Since $S$ is metrizable, [2, Corollary 2.6] yields the existence of a function $g \in \mathcal{B}_{1}(S)$ with $\|f \circ \varphi-g\|<\eta+1$.

By [14, $\S 27, \mathrm{X}], g$ has a point of continuity, and thus there exist a nonempty open set $U \subset S$ and $C \in \mathbb{R}$ such that $g>C$ on $U$. We pick $\lambda \in U$ and find $t \in(0,1)$ with $t \lambda \in U$. If $e_{n}, n \in \mathbb{N}$, denote the standard 
basic vectors in $\ell^{1}$, then $e_{n} \rightarrow 0$, and thus $t \lambda+(1-t) e_{n} \in U$ for all but finitely many $n \in \mathbb{N}$. For these indices, we obtain

$$
\begin{aligned}
C-\eta-1 & \leq g\left(t \lambda+(1-t) e_{n}\right)-\eta-1 \leq(f \circ \varphi)\left(t \lambda+(1-t) e_{n}\right) \\
& \leq t f(\varphi(\lambda))+(1-t) f\left(x_{n}\right) .
\end{aligned}
$$

This contradiction finishes the proof.

We will need the following quantitative version of [7, Proposition 2.19]. We recall that $\int^{*}$ and $\int_{*}$ denote the upper and lower integral, respectively (see [8, 133I]).

Lemma 3.5. Let $f: X \rightarrow \mathbb{R}$ be an affine function on a compact convex set $X$ and $\mu \in \mathcal{M}^{1}(X)$. Then

$$
f(r(\mu))-\operatorname{frag}(f) \leq \int_{*} f d \mu \leq \int^{*} f d \mu \leq f(r(\mu))+\operatorname{frag}(f) .
$$

Proof. If $\operatorname{frag}(f)=\infty$, the inequalities obviously hold. Otherwise we may assume by Lemma 3.4 that $f$ is bounded. Let $x$ denote the barycenter of $\mu$. We start the proof by fixing $\eta>\operatorname{frag}(f)$. We define

$$
\begin{aligned}
\mathcal{U}= & \{U \subset X: U \text { is open and there are compact convex sets } \\
& \left.K_{n} \subset X \text { such that } \mu\left(U \backslash \bigcup_{n=1}^{\infty} K_{n}\right)=0 \text { and } \operatorname{diam} f\left(K_{n}\right)<\eta\right\} .
\end{aligned}
$$

Then $V=\bigcup\{U: U \in \mathcal{U}\} \in \mathcal{U}$. Indeed, $V$ is obviously open. Since $\mu$ is inner regular with respect to compact sets, there exists a sequence $\left\{H_{k}\right\}$ of compact sets such that $\mu\left(H_{k}\right) \nearrow \mu(V)$. By compactness, we can cover each $H_{k}$ by a finite family $\left\{U_{1}, \ldots, U_{n_{k}}\right\}$ of sets contained in $\mathcal{U}$. For every $k \in \mathbb{N}$ and $U_{i}, i=1, \ldots, n_{k}$, we find a countable family of compact convex sets guaranteed by (3.1). Putting together all these families, we obtain a countable family $\mathcal{L}$ of compact convex sets which covers $\mu$-almost all of $V$ and $\operatorname{diam} f(K)<\eta$ for each $K \in \mathcal{L}$.

Our aim is to prove that $X \in \mathcal{U}$. To this end, let $\mathcal{K}$ be the family of all closed convex subsets of $X$ whose complement in $X$ is contained in $\mathcal{U}$. Let $Z$ be the intersection of $\mathcal{K}$. By the argument above, $Z$ is the smallest element of $\mathcal{K}$. Set

$$
Y=\left\{x \in Z: \operatorname{osc}_{Z} f(x) \geq \eta\right\} .
$$

(Here $\operatorname{osc}_{Z} f(x)$ denotes the oscillation of the function $\left.f\right|_{Z}$ at the point $x$.) Then $Y$ is a closed convex subset of $Z$. If $x \in Z \backslash Y$, then there exists an open convex neighborhood $U$ of $x$ such that $\bar{U} \cap Y=\emptyset$ and $\operatorname{diam} f(\bar{U} \cap Z)<\eta$. Since $U \backslash Z \in \mathcal{U}$ and $\bar{U} \cap Z$ contains $U \cap Z$, we observe that $U \in \mathcal{U}$. By the properties of $\mathcal{U}, Y$ is a closed convex subset of $Z$ whose complement in $X$ is contained in $\mathcal{U}$. By the minimality of $Z$, we have $Y=Z$. 
Then there is no open set $W \subset X$ intersecting $Z$ with $\operatorname{diam} f(W \cap Z)<\eta$. Since $\eta>\operatorname{frag}(f)$, this implies that $Z=\emptyset$. Hence $X \in \mathcal{U}$.

To finish the proof, we choose $\varepsilon>0$. Let $\left\{K_{n}\right\}$ be a sequence of compact convex subsets of $X$ such that

$$
\operatorname{diam} f\left(K_{n}\right)<\eta \quad \text { and } \quad \mu\left(X \backslash \bigcup_{n=1}^{\infty} K_{n}\right)=0 .
$$

Let $k \in \mathbb{N}$ be such that

$$
\mu\left(X \backslash\left(K_{1} \cup \cdots \cup K_{k}\right)\right)<\varepsilon
$$

and let

$$
\begin{gathered}
E_{n}=K_{n} \backslash \bigcup_{i=1}^{n-1} K_{i}, \quad n=1, \ldots, k, \quad E_{0}=X \backslash \bigcup_{n=1}^{k} K_{n}, \\
\lambda_{n}=\mu\left(E_{n}\right), \quad n=0, \ldots, k .
\end{gathered}
$$

Without loss of generality we may assume that $\lambda_{n}>0$ for $n=1, \ldots, k$. We define probability measures $\mu_{n}, n=0, \ldots, k$, by

$$
\mu_{n}= \begin{cases}\left.\frac{1}{\lambda_{n}} \mu\right|_{E_{n}} & \text { if } \lambda_{n}>0 \\ \varepsilon_{x} & \text { if } \lambda_{n}=0 .\end{cases}
$$

Let $x_{n}$ be the barycenter of $\mu_{n}, n=0, \ldots, k$. Then $x_{n} \in \overline{\mathrm{co}} E_{n} \subset K_{n}$, $n=1, \ldots, k$. Obviously,

$$
\sum_{n=0}^{N} \lambda_{n}=1, \quad \sum_{n=0}^{k} \lambda_{n} x_{n}=x, \quad \sum_{n=0}^{k} \lambda_{n} \mu_{n}=\mu,
$$

and

$$
f\left(x_{0}\right)-2\|f\| \leq \int_{*} f d \mu_{0} \leq \int^{*} f d \mu_{0} \leq f\left(x_{0}\right)+2\|f\| .
$$

Since diam $f\left(K_{n}\right)<\eta$, from $(3.2)-(3.4)$ we obtain

$$
\begin{aligned}
\int^{*} f d \mu & =\lambda_{0} \int^{*} f d \mu_{0}+\sum_{n=1}^{k} \lambda_{n} \int^{*} f d \mu_{n} \\
& \leq \lambda_{0}\left(f\left(x_{0}\right)+2\|f\|\right)+\sum_{n=1}^{k} \int_{E_{n}}^{*} f d \mu \\
& \leq \lambda_{0}\left(f\left(x_{0}\right)+2\|f\|\right)+\sum_{n=1}^{k} \int_{E_{n}}\left(f\left(x_{n}\right)+\eta\right) d \mu \\
& =\lambda_{0}\left(f\left(x_{0}\right)+2\|f\|\right)+\sum_{n=1}^{k} \lambda_{n}\left(f\left(x_{n}\right)+\eta\right) \\
& \leq f\left(\sum_{n=0}^{k} \lambda_{n} x_{n}\right)+\eta+\varepsilon 2\|f\|=f(x)+\eta+\varepsilon 2\|f\| .
\end{aligned}
$$


Letting $\varepsilon \rightarrow 0$, we obtain $\int^{*} f d \mu \leq f(x)+\eta$. Since $\eta$ is arbitrary, $\int^{*} f d \mu \leq$ $f(x)+\operatorname{frag}(f)$. Analogously we obtain the reverse inequality $f(x)-\operatorname{frag}(f) \leq$ $\int_{*} f d \mu$, which concludes the proof.

\section{Proofs of the main results}

Proof of Theorem 1.4. Let $f: K \rightarrow E$ be a mapping. To verify (a), we notice that the proof of the inequality

$$
\frac{1}{2} \sigma-\operatorname{frag}_{c}(f) \leq \operatorname{dist}\left(f, \mathcal{B}_{1}(X, E)\right)
$$

in [2, Theorem 2.5] does not require any assumption on $X$ (here $\sigma-\operatorname{frag}_{c}(f)$ is the index of $\sigma$-fragmentability defined in [2, Definition 1]). By [2, Theorem 2.1], $\sigma-\operatorname{frag}_{c}(f)=\operatorname{frag}(f)$ for hereditarily Baire spaces and thus assertion (a) follows.

For the proof of (b), assume that $f$ is Baire measurable. We use [10, Theorem 1] to deduce that the range $f(K)$ is $K$-analytic, and thus separable. Hence there exists $\alpha \in\left(0, \omega_{1}\right)$ such that $f$ is $\Sigma_{\alpha+1}(\operatorname{Baire}(K))$-measurable. By [22, Corollary 5.5], $f$ is a mapping of Baire class $\alpha$ (i.e., $f \in \mathcal{C}_{\alpha}(K, E)$ ). It follows that there exists a countable family $\mathcal{F}=\left\{f_{n}: n \in \mathbb{N}\right\} \subset \mathcal{C}(K, E)$ such that $f \in \mathcal{F}_{\alpha}$.

(We recall the following notation from [22, Definition 2.4]. If $\mathcal{F}$ is a family of mappings from a set $X$ to a topological space $Y$, we inductively define Baire classes generated by $\mathcal{F}$ as follows: Let $\mathcal{F}_{0}=\mathcal{F}$ and for each countable ordinal $\alpha \in\left(0, \omega_{1}\right)$, let $\mathcal{F}_{\alpha}$ be the family of all pointwise limits of sequences from $\bigcup_{\beta<\alpha} \mathcal{F}_{\beta}$.)

Let $\varphi: K \rightarrow E^{\mathbb{N}}$ be defined by

$$
\varphi(x)=\left\{f_{n}(x)\right\}_{n \in \mathbb{N}}, \quad x \in K .
$$

Then $L=\varphi(K)$ is a compact metrizable space. Since, for $x_{1}, x_{2} \in K$, $f_{n}\left(x_{1}\right)=f_{n}\left(x_{2}\right)$ for each $n \in \mathbb{N}$ implies $f\left(x_{1}\right)=f\left(x_{2}\right)$, there exists a mapping $g: L \rightarrow E$ such that $f=g \circ \varphi$. By Lemma 3.1, frag $(f)=\operatorname{frag}(g)$. By [2, Theorem 2.5], for every $\eta>\operatorname{frag}(g)$, there exists a function $h \in \mathcal{B}_{1}(L, E)$ such that $\|g-h\|<\eta$. Then $\|f-h \circ \varphi\|<\eta$, and hence $\operatorname{dist}\left(f, \mathcal{B}_{1}(K, E)\right)<\eta$. Since $\eta>\operatorname{frag}(f)$ is arbitrary, $\operatorname{dist}\left(f, \mathcal{B}_{1}(K, E)\right) \leq \operatorname{frag}(f)$.

If $f$ is Baire measurable and $E=\mathbb{R}$ then we proceed as in the proof of (b) and obtain, from Lemma 3.1 and [2, Theorem 2.5],

$$
\frac{1}{2} \operatorname{frag}(f)=\frac{1}{2} \operatorname{frag}(g)=\operatorname{dist}\left(g, \mathcal{B}_{1}(Y)\right) \geq \operatorname{dist}\left(f, \mathcal{B}_{1}(X)\right) .
$$

This concludes the proof.

THEOREM 4.1. There exists a metrizable simplex $X$ with the following property: for any $\varepsilon>0$ there exists a strongly affine function $f: X \rightarrow[-1,1]$ such that $\operatorname{frag}(f)<\varepsilon$ and $\operatorname{dist}\left(f, \mathfrak{A}_{1}(X)\right) \geq 1 / 2$. 
Proof. For each natural number $m>1$, let $\left(K_{m}, \mathcal{H}_{m}\right)$ be the function space from Section 2 and let $f_{m}: K_{m} \rightarrow[-1,1]$ be the function from Definition 2.2. Let $K=\bigcup_{m=2}^{\infty} K_{m} \cup\left\{x_{\infty}\right\}$ be the one-point compactification of the topological union of the spaces $K_{m}$ and let

$$
\mathcal{H}=\left\{f \in \mathcal{C}(K):\left.f\right|_{K_{m}} \in \mathcal{H}_{m}, m>1\right\} .
$$

It is easy to verify that $(K, \mathcal{H})$ is a simplicial function space with $\mathrm{Ch}_{\mathcal{H}} K=$ $\left\{x_{\infty}\right\} \cup \bigcup_{m=2}^{\infty} \mathrm{Ch}_{\mathcal{H}_{m}} K_{m}$ and $\mathcal{A}_{c}(\mathcal{H})=\mathcal{H}$. If $X$ denotes the state space of $\mathcal{H}$, we obtain a metrizable simplex (see [23, Theorem 2.6(a)]). We claim that $X$ has the required property.

To see this, we fix $\varepsilon>0$. Let $m>1$ be a natural number satisfying $2(2 m+1)^{-1}<\varepsilon$. If $f_{m}: K_{m} \rightarrow[-1,1]$ is as in Definition 2.2, we define $f: K \rightarrow[-1,1]$ as

$$
f(x)= \begin{cases}f_{m}(x), & x \in K_{m}, \\ 0, & \text { otherwise. }\end{cases}
$$

Let $I: \mathcal{U}^{b}(K) \cap \mathcal{H}^{\perp \perp} \rightarrow \mathfrak{A}_{\mathrm{bf}}(X)$ be the identification from 1.1). Since $\operatorname{frag}(f)=2(2 m+1)^{-1}, \operatorname{frag}(I f)<\varepsilon$ by Lemma 3.3. If $g$ is any function in $\mathfrak{A}_{1}(X)$, it follows from [23, Theorem 2.5(f)] that $I^{-1} g \in \mathcal{B}_{1}^{b}(K) \cap \mathcal{H}^{\perp \perp}$. Then $\left.I^{-1} g\right|_{K_{m}} \in \mathcal{B}_{1}^{b}\left(K_{m}\right) \cap \mathcal{H}_{m}^{\perp \perp}$, and thus $\left\|f-I^{-1} g\right\| \geq 1 / 2$ by Lemma 2.3(d). Hence $\|I f-g\| \geq 1 / 2$, and the proof is complete.

Proof of Theorem 1.1. Let $(K, \mathcal{H})$ be the simplicial function space constructed in the proof of Theorem 4.1 and let $X$ be the state space of $\mathcal{H}$. Then $\mathcal{H}$ is isometrically isomorphic to $\mathfrak{A}^{c}(X)$ via the mapping $I$, and thus it is a separable $L_{1}$-predual (see [7, Proposition 3.23]). Given $\varepsilon>0$, let $m>1$ be a natural number with $(2 m+1)^{-1}<\varepsilon$ and let $f: K \rightarrow[-1,1]$ be the function from 4.1). If $\pi: B_{\mathcal{M}(K)} \rightarrow B_{\mathcal{H}^{*}}$ is the restriction mapping, let $\widehat{f}: B_{\mathcal{H}^{*}} \rightarrow[-1,1]$ be defined as

$$
\widehat{f}(s)=\mu(f), \quad \pi(\mu)=s, \quad s \in B_{\mathcal{H}^{*}} .
$$

Obviously, $\widehat{f}$ is a restriction of an element from $\mathcal{H}^{* *}$ to $B_{\mathcal{H}^{*}}$. By Lemmas 3.2 and 3.1 ,

$$
\operatorname{dist}\left(\widehat{f}, \mathcal{B}_{1}\left(B_{E^{*}}\right)\right)=\frac{1}{2} \operatorname{frag}(\widehat{f})=(2 m+1)^{-1}<\varepsilon .
$$

By [23, Theorem 2.5(a)], $\widehat{f} \in \mathfrak{A}_{\mathrm{bf}}\left(B_{\mathcal{H}^{*}}\right)$. Finally,

$$
\frac{1}{2} \leq \operatorname{dist}\left(I f, \mathfrak{A}_{1}(\mathbf{S}(\mathcal{H}))\right) \leq \operatorname{dist}\left(\widehat{f}, \mathfrak{A}_{1}\left(B_{\mathcal{H}^{*}}\right)\right) .
$$

This concludes the proof.

Proof of Theorem 1.2. Let $E$ be an $L_{1}$-predual such that ext $B_{E^{*}}$ is a Lindelöf $H$-set and let $f: B_{E^{*}} \rightarrow \mathbb{R}$ be the restriction of an element $x^{* *} \in$ $E^{* *}$. By [17, Theorem], there exists a simplex $X$, an isometric embedding $j: E \rightarrow \mathfrak{A}^{c}(X)$ and a projection $P: \mathfrak{A}^{c}(X) \rightarrow j(E)$ of norm 1. Moreover, 
if $E$ is separable, $X$ can be chosen to be metrizable. Further, it is proved in [17, Corollary III] that there exists an affine continuous surjection $\varphi$ : $X \rightarrow B_{E^{*}}$ such that

(1) $\varphi(\operatorname{ext} X)=\operatorname{ext} B_{E^{*}} \cup\{0\}$ and $\varphi^{-1}\left(\operatorname{ext} B_{E^{*}}\right) \subset \operatorname{ext} X$,

(2) $\left.\varphi\right|_{\operatorname{ext} X}$ is injective,

(3) ext $X \backslash \varphi^{-1}$ (ext $\left.B_{E^{*}}\right)$ is a singleton,

(4) $j(e)(x)=\left(\left.e\right|_{B_{E^{*}}} \circ \varphi\right)(x), e \in E, x \in X$.

(In the notation of 17, the embedding $j$ is denoted by $T$ and $\varphi$ is denoted by $q$. Conditions (1), (2) and (3) are explicitly stated in [17, Corollary III], condition (4) follows from the definitions of $T$ on p. 175 and $q$ on p. 176.)

We claim that ext $X$ is a Lindelöf $H$-set. To show this, we first observe that ext $X$ differs from the $H$-set $\varphi^{-1}\left(\operatorname{ext} B_{E^{*}}\right)$ by a singleton (see (1) and (3)), and thus it is an $H$-set. Second, let $F \subset X \backslash$ ext $X$ be a compact set. By (1), $\varphi(F)$ is disjoint from ext $B_{E^{*}}$. Since ext $B_{E^{*}}$ is Lindelöf, 24, Lemma 14] provides an $F_{\sigma}$ set $A$ with

$$
\operatorname{ext} B_{E^{*}} \subset A \subset \operatorname{ext} B_{E^{*}} \backslash \varphi(F) .
$$

If $x_{0} \in X$ is the singleton ext $X \backslash \varphi^{-1}\left(\operatorname{ext} B_{E^{*}}\right)$, then $\varphi^{-1}(A)$ is an $F_{\sigma}$ set in $X$ satisfying

$$
\operatorname{ext} X \subset \varphi^{-1}(A) \cup\left\{x_{0}\right\} \subset X \backslash F .
$$

By [24, Lemma 15], ext $X$ is a Lindelöf space.

If $f$ is a Baire measurable function on $B_{E^{*}}$, then $f \circ \varphi$ is Baire measurable on $X$. If $E$ is separable, $X$ is metrizable. In both cases, Lemma 3.1 and Theorem 1.4 give

$$
\operatorname{dist}\left(f \circ \varphi, \mathcal{B}_{1}(X)\right)=\frac{1}{2} \operatorname{frag}(f \circ \varphi)=\frac{1}{2} \operatorname{frag}(f)=\operatorname{dist}\left(f, \mathcal{B}_{1}\left(B_{E^{*}}\right)\right) .
$$

We fix $\eta>\operatorname{dist}\left(f, \mathcal{B}_{1}\left(B_{E^{*}}\right)\right)$. Let $g \in \mathcal{B}_{1}(X)$ satisfy $\|f \circ \varphi-g\|<\eta$. Without loss of generality we may assume that $\|f \circ \varphi\|=\|g\|$. By [24, Theorem 1], there exists a function $h \in \mathfrak{A}_{1}(X)$ such that $h=g$ on ext $X$ and $\|h\|=\|g\|$.

We claim that $\|h-f \circ \varphi\| \leq 3 \eta$. To this end, let $x \in X$ be given. We find a maximal measure $\mu \in \mathcal{M}^{1}(X)$ with $r(\mu)=x$ (see [1, Proposition I.2.1]). If $f$ is Baire measurable, the set

$$
F=\{x \in X:|h(x)-(f \circ \varphi)(x)| \leq \eta\}
$$

is a Baire set in $X$ containing ext $X$. By [1, Corollary I.4.12 and the subsequent Remark], $\mu(X \backslash F)=0$. Hence, by Lemma 3.5 .

$$
h(x)=\mu(h)=\int_{F} h d \mu \leq \int_{F}^{*} f \circ \varphi d \mu+\eta \leq(f \circ \varphi)(x)+\eta+2 \eta .
$$


If $E$ is separable, $X$ is metrizable, and thus $\mu(X \backslash \operatorname{ext} X)=0$ (see [1, Corollary I.4.12]). As above we obtain

$$
\mu(h)=\int_{\operatorname{ext} X} h d \mu \leq \int_{\operatorname{ext} X}^{*} f \circ \varphi d \mu \leq(f \circ \varphi)(x)+3 \eta .
$$

Analogously,

$$
h(x) \geq(f \circ \varphi)(x)-3 \eta .
$$

Thus $\|h-f \circ \varphi\| \leq 3 \eta$.

Since $P: \mathfrak{A}^{c}(X) \rightarrow j(E)$ is a projection of norm 1 , to any $x \in X$ we can assign a measure $\mu_{x} \in B_{\mathcal{M}(X)}$ such that

$$
\operatorname{Pf}(x)=\mu_{x}(f), \quad f \in \mathfrak{A}^{c}(X) .
$$

Since $P$ is identity on $j(E)$, we obtain

$$
\mu_{x}\left(\left.e\right|_{B_{E^{*}}} \circ \varphi\right)=\left(\left.e\right|_{B_{E^{*}}} \circ \varphi\right)(x), \quad x \in X, e \in E .
$$

We use equality (4.2) to extend the domain of $P$ to any bounded universally measurable function on $X$.

We claim that

$$
\left|\mu_{x}(h)-f(\varphi(x))\right| \leq 5 \eta, \quad x \in X .
$$

To verify this, let $x \in X$ be given. We write

$$
\mu_{x}=a_{1} \mu_{1}-a_{2} \mu_{2}, \quad a_{1}, a_{2} \geq 0 \text { with } a_{1}+a_{2} \leq 1, \mu_{1}, \mu_{2} \in \mathcal{M}^{1}(X),
$$

and let $x_{1}, x_{2} \in X$ be the barycenters of $\mu_{1}, \mu_{2}$, respectively. Then

$$
\varphi(x)=a_{1} \varphi\left(x_{1}\right)-a_{2} \varphi\left(x_{2}\right) .
$$

Indeed, if $e \in E$ is arbitrary, let $\widehat{e}$ denote its restriction to $B_{E^{*}}$. Let $\varphi_{\sharp}$ : $\mathcal{M}^{1}(X) \rightarrow \mathcal{M}^{1}\left(B_{E^{*}}\right)$ denote the mapping induced by $\varphi: X \rightarrow B_{E^{*}}$ (see [9, Theorems 418I and 418L]). Then

$$
\begin{aligned}
\widehat{e}(\varphi(x)) & =\mu_{x}(\widehat{e} \circ \varphi)=a_{1} \mu_{1}(\widehat{e} \circ \varphi)-a_{2} \mu_{2}(\widehat{e} \circ \varphi) \\
& =a_{1}\left(\varphi_{\sharp} \mu_{1}\right)(\widehat{e})-a_{2}\left(\varphi_{\sharp} \mu_{2}\right)(\widehat{e})=a_{1} \mu_{1}(\widehat{e} \circ \varphi)-a_{2} \mu_{2}(\widehat{e} \circ \varphi) \\
& =a_{1} \widehat{e}\left(\varphi\left(x_{1}\right)\right)-a_{2} \widehat{e}\left(\varphi\left(x_{2}\right)\right)=\widehat{e}\left(a_{1} \varphi\left(x_{1}\right)-a_{2} \varphi\left(x_{2}\right)\right) .
\end{aligned}
$$

Hence (4.4) holds.

Further, by Lemma 3.5 .

$$
\begin{aligned}
\mu_{1}(h) & \leq \int_{X}^{*} f \circ \varphi d \mu_{1}+3 \eta=\int_{B_{E^{*}}}^{*} f d\left(\varphi_{\sharp} \mu_{1}\right)+3 \eta \\
& \leq f\left(r\left(\varphi_{\sharp} \mu_{1}\right)\right)+3 \eta+2 \eta=f\left(\varphi\left(x_{1}\right)\right)+5 \eta .
\end{aligned}
$$

Analogously,

$$
\mu_{1}(h) \geq f\left(\varphi\left(x_{1}\right)\right)-5 \eta
$$

Hence

$$
\left|\mu_{1}(h)-f\left(\varphi\left(x_{1}\right)\right)\right| \leq 5 \eta \text {. }
$$


Similarly we obtain

$$
\left|\mu_{2}(h)-f\left(\varphi\left(x_{2}\right)\right)\right| \leq 5 \eta .
$$

By combining these inequalities and (4.4) we have

$$
\begin{aligned}
\left|\mu_{x}(h)-f(\varphi(x))\right| & =\left|a_{1} \mu_{1}(h)-a_{2} \mu_{2}(h)-f\left(a_{1} \varphi\left(x_{1}\right)-a_{2} \varphi\left(x_{2}\right)\right)\right| \\
& =\left|a_{1}\left(\mu_{1}(h)-f\left(\varphi\left(x_{1}\right)\right)\right)-a_{2}\left(\mu_{2}(h)-f\left(\varphi\left(x_{2}\right)\right)\right)\right| \\
& \leq 5 \eta\left(a_{1}+a_{2}\right)=5 \eta .
\end{aligned}
$$

This gives 4.3.

If $\left\{h_{n}\right\}$ is a bounded sequence in $\mathfrak{A}^{c}(X)$ pointwise converging to $h$, the Lebesgue bounded convergence theorem implies that $P h_{n} \rightarrow P h$. Since $P h_{n} \in j(E)$, there exist elements $e_{n} \in E, n \in \mathbb{N}$, such that

$$
P h_{n}=\left.e_{n}\right|_{B_{E^{*}}} \circ \varphi, \quad n \in \mathbb{N} .
$$

Then $\left\{\left.e_{n}\right|_{B_{E^{*}}}\right\}$ converges to a function $e \in \mathfrak{A}_{1}\left(B_{E^{*}}\right)$. It follows that $P h=$ $\left.e\right|_{B_{E^{*}}} \circ \varphi$ and, by 4.3 ,

$$
\left\|\left.e\right|_{B_{E^{*}}}-f\right\|=\left\|\left.e\right|_{B_{E^{*}}} \circ \varphi-f \circ \varphi\right\|=\|P h-f \circ \varphi\| \leq 5 \eta .
$$

Hence $\operatorname{dist}\left(f, \mathfrak{A}_{1}\left(B_{E^{*}}\right)\right) \leq 5 \eta$. Since $\eta>\operatorname{dist}\left(f, \mathcal{B}_{1}\left(B_{E^{*}}\right)\right)$ is arbitrary, we obtain

$$
\operatorname{dist}\left(f, \mathfrak{A}_{1}\left(B_{E^{*}}\right)\right) \leq 5 \operatorname{dist}\left(f, \mathcal{B}_{1}\left(B_{E^{*}}\right)\right) .
$$

TheOREM 4.2. Let $X$ be a compact convex set such that $\mathfrak{A}^{c}(X)$ does not contain $\ell^{1}$ and $f: X \rightarrow \mathbb{R}$ be an affine function. Then $\operatorname{dist}\left(f, \mathfrak{A}_{1}(X)\right)$ $\leq 2 \operatorname{dist}\left(f, \mathcal{B}_{1}(X)\right)$.

Proof. If $\operatorname{dist}\left(f, \mathcal{B}_{1}(X)\right)=\infty$, the assertion obviously holds. We assume that $\operatorname{dist}\left(f, \mathcal{B}_{1}(X)\right)<\infty$ and fix $\eta>\operatorname{dist}\left(f, \mathcal{B}_{1}(X)\right)$. By Theorem 1.4 and Lemma 3.4, $f$ is bounded. We find a function $g \in \mathcal{B}_{1}(X)$ such that $\|f-g\|<\eta$. Without loss of generality we may assume that $\|g\|=\|f\|$. It is easy to find (see e.g. [16, Exercise 3.G.1]) sequences $\left\{u_{n}\right\}$ and $\left\{l_{n}\right\}$ of functions on $X$ such that every $u_{n}$ is upper semicontinuous, every $l_{n}$ is lower semicontinuous and

$$
-\|g\| \leq u_{n} \nearrow g, \quad\|g\| \geq l_{n} \searrow g .
$$

We fix $n \in \mathbb{N}$ and $x \in X$. By [1, Corollary I.3.6], there exist measures $\mu_{1}, \mu_{2} \in \mathcal{M}^{1}(X)$ representing $x$ such that

$$
\left(u_{n}-\eta\right)^{*}(x)=\mu_{1}\left(u_{n}-\eta\right) \text { and }\left(l_{n}+\eta\right)_{*}(x)=\mu_{2}\left(l_{n}+\eta\right) .
$$

(We recall that $f^{*}$ and $f_{*}$ are the upper and lower envelopes of a function $f$, respectively; see [1, p. 4].) By [11, Theorem 4.2], $f$ is universally measurable and $\mu(f)=f(r(\mu))$ for every $\mu \in \mathcal{M}^{1}(X)$. (Here we use the identification 
of $\mathfrak{A}^{b}(X)$ with $\left(\mathfrak{A}^{c}(X)\right)^{* *}$.) Hence

$$
\begin{gathered}
\left(u_{n}-\eta\right)^{*}(x)=\mu_{1}\left(u_{n}-\eta\right)<\int f d \mu_{1}=f(x), \\
\left(l_{n}+\eta\right)_{*}(x)=\mu_{2}\left(l_{n}+\eta\right)>\int f d \mu_{2}=f(x) .
\end{gathered}
$$

Since the upper envelope is an upper semicontinuous concave function and the lower envelope is a lower semicontinuous convex function (see [1, p. 4]), the Hahn-Banach theorem provides a function $h_{n} \in \mathfrak{A}^{c}(X)$ such that

$$
\left(u_{n}-\eta\right)^{*}<h_{n}<\left(l_{n}+\eta\right)_{*} .
$$

Since $\mathfrak{A}^{c}(X)$ does not contain $\ell^{1}$, Rosenthal's theorem (see [12, p. 18]) provides a subsequence $\left\{h_{n_{k}}\right\}$ of $\left\{h_{n}\right\}$ that converges pointwise to a function $h$. Then $h \in \mathfrak{A}_{1}(X)$ and

$$
g-\eta=\lim _{k \rightarrow \infty} u_{n_{k}}-\eta \leq h \leq \lim _{k \rightarrow \infty} l_{n_{k}}+\eta=g+\eta .
$$

Since $\|g-f\|<\eta$, we obtain

$$
\|f-h\|<2 \eta
$$

Since $\eta>\operatorname{dist}\left(f, \mathcal{B}_{1}(X)\right)$ is arbitrary, the proof is finished.

Proof of Theorem 1.3. This follows from Theorem 4.2.

Acknowledgements. The author would like to thank his colleagues at the Faculty of Mathematics and Physics for a substantial help during the work on the paper. He would also like to thank the referee for several helpful comments.

The author was supported in part by the grant GAAV IAA 100190901 and in part by the Research Project MSM 0021620839 from the Czech Ministry of Education.

\section{References}

[1] E. M. Alfsen, Compact Convex Sets and Boundary Integrals, Ergeb. Math. Grenzgeb. 57, Springer, New York, 1971.

[2] C. Angosto, B. Cascales, and I. Namioka, Distances to spaces of Baire one functions, Math. Z. 263 (2009), 103-124.

[3] S. A. Argyros, G. Godefroy, and H. P. Rosenthal, Descriptive set theory and Banach spaces, in: Handbook of the Geometry of Banach Spaces, Vol. 2, North-Holland, Amsterdam, 2003, 1007-1069.

[4] L. Asimow and A. J. Ellis, Convexity Theory and its Applications in Functional Analysis, London Math. Soc. Monogr. 16, Academic Press, London, 1980.

[5] B. Cascales, W. Marciszewski, and M. Raja, Distance to spaces of continuous functions, Topology Appl. 153 (2006), 2303-2319.

[6] G. Choquet, Lectures on Analysis. Vol. I-III: Infinite Dimensional Measures and Problem Solutions, W. A. Benjamin, New York, 1969. 
[7] V. P. Fonf, J. Lindenstrauss, and R. R. Phelps, Infinite dimensional convexity, in: Handbook of the Geometry of Banach Spaces, Vol. I, North-Holland, Amsterdam, 2001, 599-670.

[8] D. H. Fremlin, Measure Theory. Vol. 1, Torres Fremlin, Colchester, 2004.

[9] —, Measure Theory. Vol. 4, Torres Fremlin, Colchester, 2006.

[10] Z. Frolík, A measurable map with analytic domain and metrizable range is quotient, Bull. Amer. Math. Soc. 76 (1970), 1112-1117.

[11] R. Haydon, Some more characterizations of Banach spaces containing $l_{1}$, Math. Proc. Cambridge Philos. Soc. 80 (1976), 269-276.

[12] W. B. Johnson and J. Lindenstrauss, Basic concepts in the geometry of Banach spaces, in: Handbook of the Geometry of Banach Spaces, Vol. I, North-Holland, Amsterdam, 2001, 1-84.

[13] U. Krause, Der Satz von Choquet als ein abstrakter Spektralsatz und vice versa, Math. Ann. 184 (1970), 275-296.

[14] C. Kuratowski, Topologie. Vol. I, 4th ed., Monografie Mat. 20, Państwowe Wydawnictwo Naukowe, Warszawa, 1958.

[15] H. E. Lacey, The Isometric Theory of Classical Banach Spaces, Grundlehren Math. Wiss. 208. Springer, New York, 1974.

[16] J. Lukeš, J. Malý, and L. Zajíček, Fine Topology Methods in Real Analysis and Potential Theory, Lecture Notes in Math. 1189, Springer, Berlin, 1986.

[17] W. Lusky, Every $L_{1}$-predual is complemented in a simplex space, Israel J. Math. 64 (1988), 169-178.

[18] E. Odell and H. P. Rosenthal, A double-dual characterization of separable Banach spaces containing $l^{1}$, ibid. 20 (1975), 375-384.

[19] R. R. Phelps, Lectures on Choquet's Theorem, 2nd ed., Lecture Notes in Math. 1757, Springer, Berlin, 2001.

[20] M. Rogalski, Opérateurs de Lion, projecteurs boréliens et simplexes analytiques, J. Funct. Anal. 2 (1968), 458-488.

[21] Z. Semadeni, Banach Spaces of Continuous Functions. Vol. I, Monografie Mat. 55, PWN-Polish Sci. Publ., Warszawa, 1971.

[22] J. Spurný, Baire and Borel functions in topological spaces, Acta Math. Hungar., to appear.

[23] —, Baire classes of Banach spaces and strongly affine functions, Trans. Amer. Math. Soc. 362 (2010), 1659-1680.

[24] J. Spurný and O. F. K. Kalenda, A solution of the abstract Dirichlet problem for Baire-one functions, J. Funct. Anal. 232 (2006), 259-294.

[25] M. Talagrand, Sur les convexes compacts dont l'ensemble des points extrémaux est $\mathcal{K}$-analytique, Bull. Soc. Math. France 107 (1979), 49-53.

Jiří Spurný

Department of Mathematical Analysis

Faculty of Mathematics and Physics

Charles University

Sokolovská 83

18675 Praha 8, Czech Republic

E-mail: spurny@karlin.mff.cuni.cz 